\title{
Tailored 2D/3D Halide Perovskite Heterointerface for Substantially Enhanced Endurance in Conducting Bridge Resistive Switching Memory
}

SangMyeong Lee ${ }^{\dagger}$, Hyojung Kim ${ }^{\ddagger}$, Dong Hoe Kim ${ }^{\S}$, Won Bin $\mathrm{Kim}^{\dagger}$, Jae Myeong Lee ${ }^{\dagger}$, Jaeho Choi ${ }^{\ddagger}$, Hyunjung Shin", Gill Sang Han*,†, Ho Won Jang ${ }^{*, \dagger}$ and Hyun Suk Jung ${ }^{*, \dagger}$

${ }^{\dagger}$ School of Advanced Materials Science and Engineering, Sungkyunkwan University, Suwon 16419, Republic of Korea

* Department of Materials Science and Engineering, Seoul National University, Seoul 08826, Republic of Korea

$\S$ Department of Nanotechnology \& Advanced Materials Engineering Sejong University, Seoul, 05006, Republic of Korea

"Department of Energy Science, Sungkyunkwan University, Suwon 16419, Republic of Korea

*E-mail: hsjung1@skku.edu, hwjang@snu.ac.kr, hangillsang1@gmail.com 


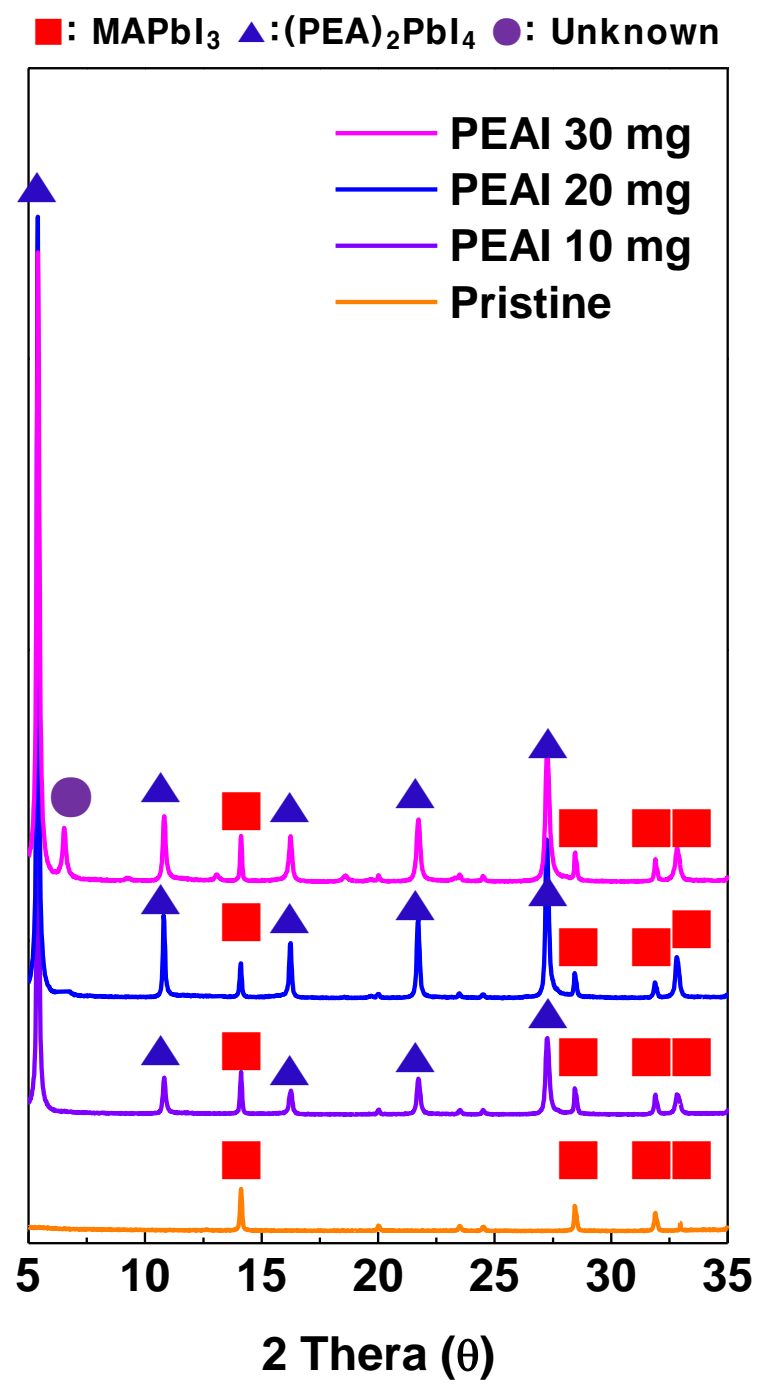

Figure S1. XRD patterns of the 2D/3D perovskite films fabricated using different concentrations of PEAI solution. 
a

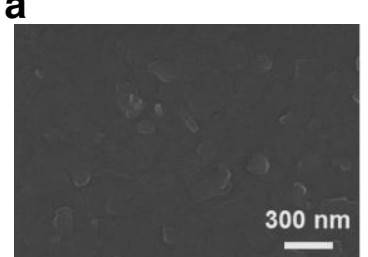

b

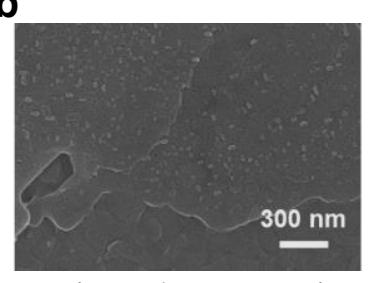

C

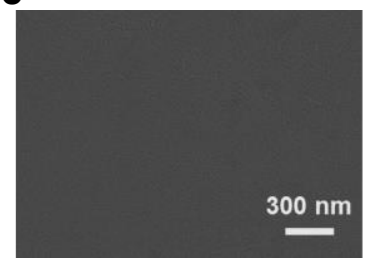

d

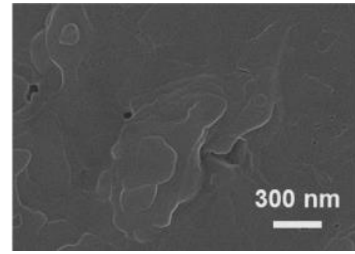

Figure S2. Plane view scanning electron microscopy (SEM) images of: (a) pristine 3D perovskite, (b) $10 \mathrm{mg}$ PEAI, (c) $20 \mathrm{mg}$ PEAI, and (d) $30 \mathrm{mg}$ PEAI. 

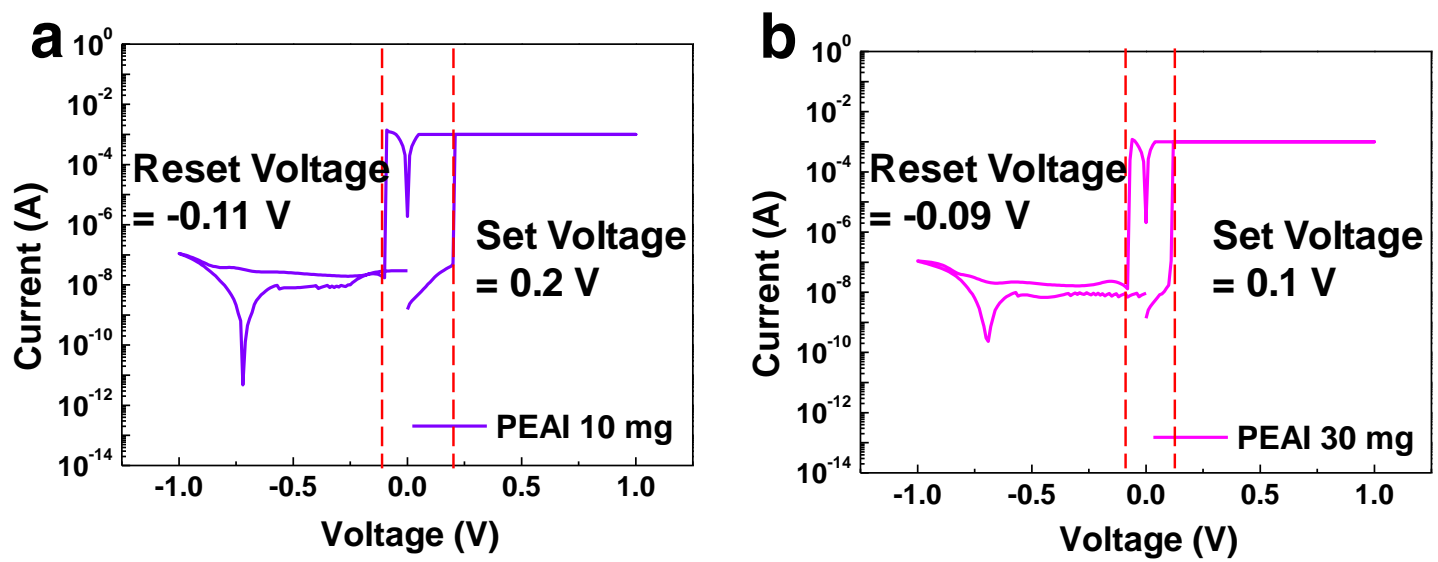

Figure S3. Typical current-voltage $(I-V)$ characteristics of HP-based RS devices. (a) PEAI 10 mg (b) PEAI 30 mg. 


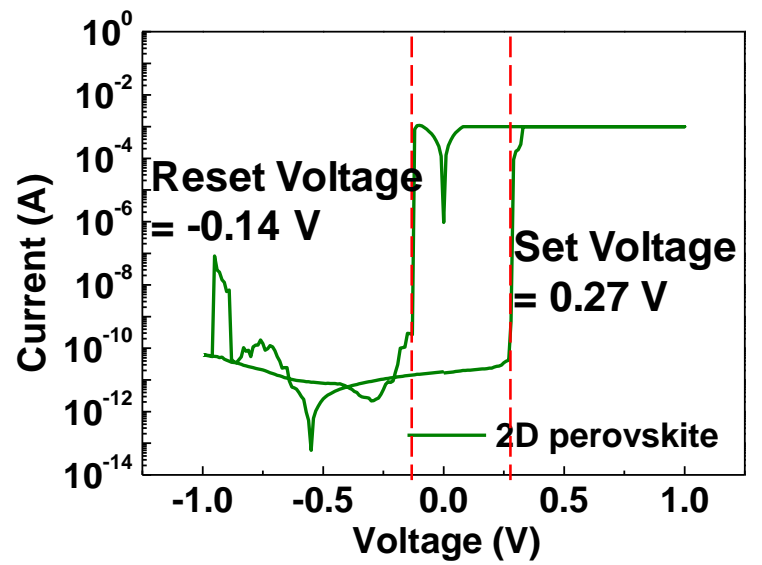

Figure S4. Typical $I-V$ characteristics of 2D HP-based RS devices. 

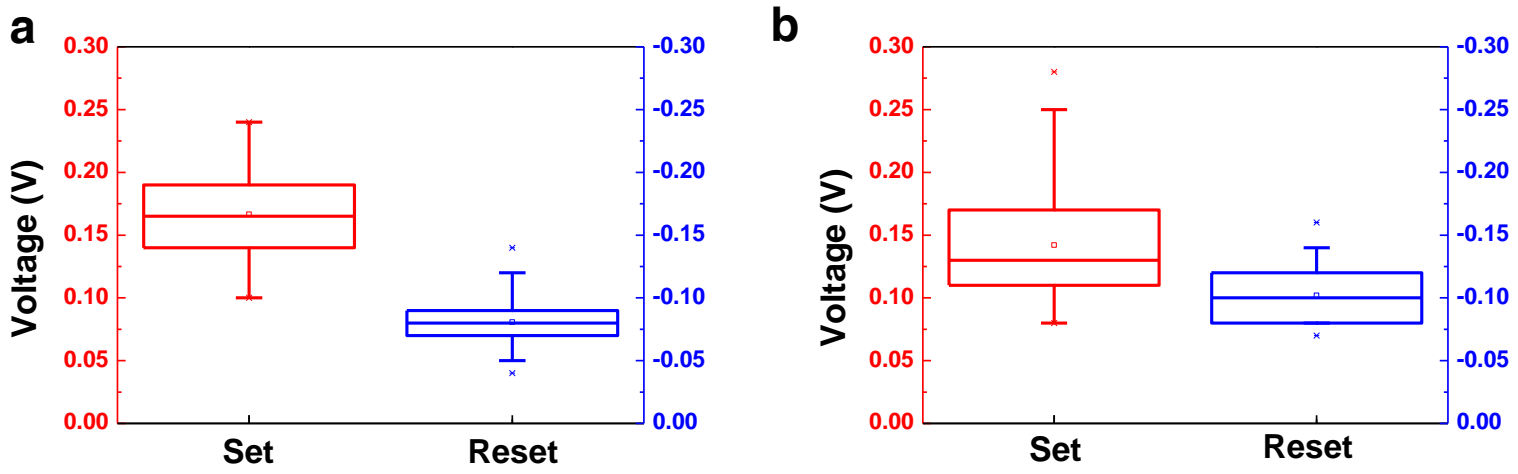

Figure S5. Average of set and reset voltage. (a) 3D perovskite and (b) 2D/3D perovskite 

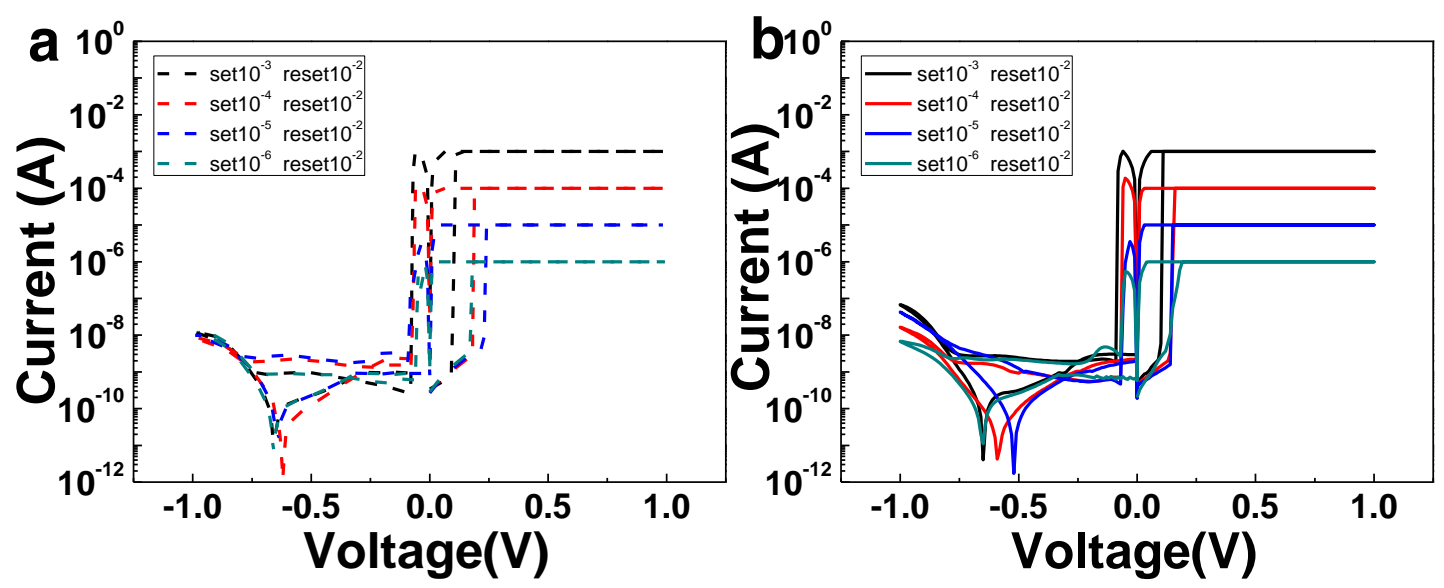

Figure S6. $I-V$ characteristics exhibiting the potential for multilevel switching (a) $I-V$ characteristics of $3 \mathrm{D}$ perovskite and (b) $I-V$ characteristics of $2 \mathrm{D} / 3 \mathrm{D}$ perovskite (compliance current $=10^{-3}, 10^{-4}, 10^{-5}$, and $10^{-6} \mathrm{~A}$ ). 

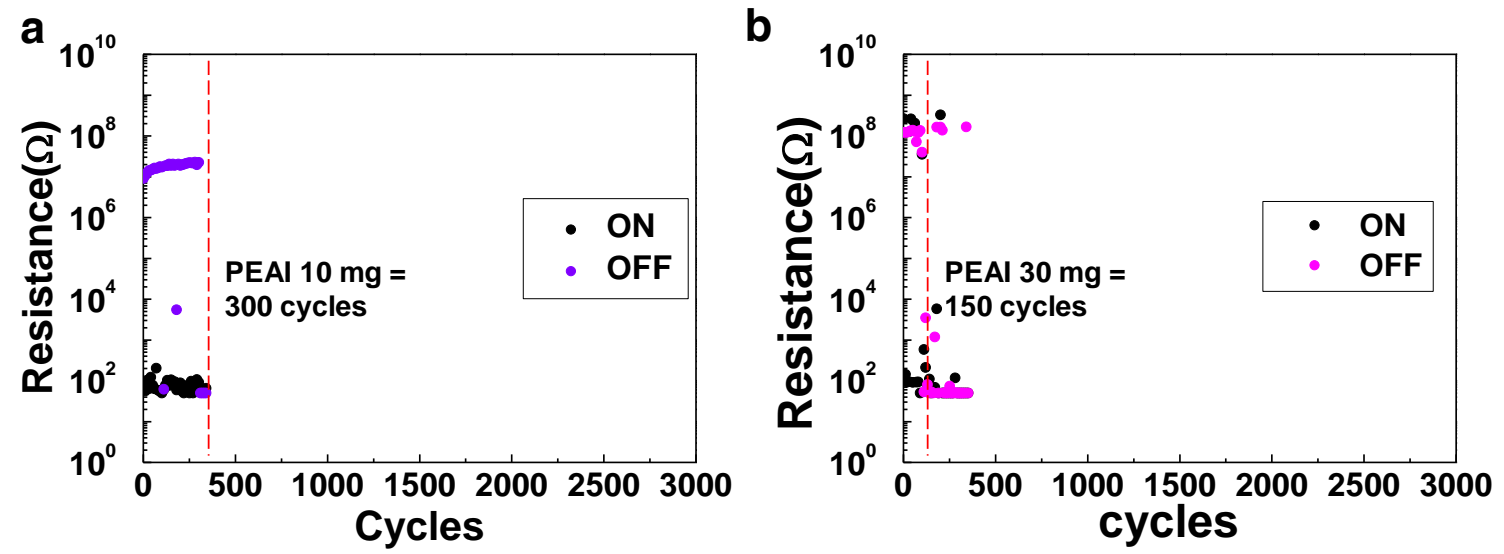

Figure S7. RS behavior measured with the DC pulses. Pulse duration is fixed at $640 \mu$ s. (a) PEAI $10 \mathrm{mg}$ (b) PEAI $30 \mathrm{mg}$. 


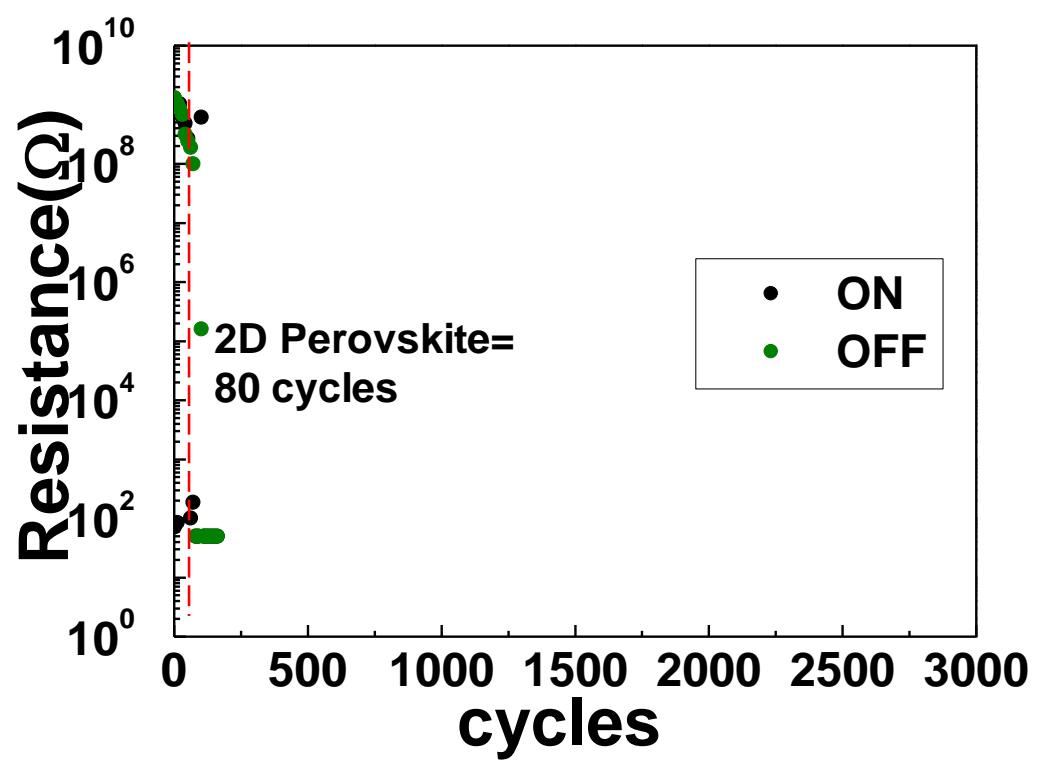

Figure S8. 2D perovskite based RS behavior measured with the DC pulses. Pulse duration is fixed at $640 \mu$ s. 


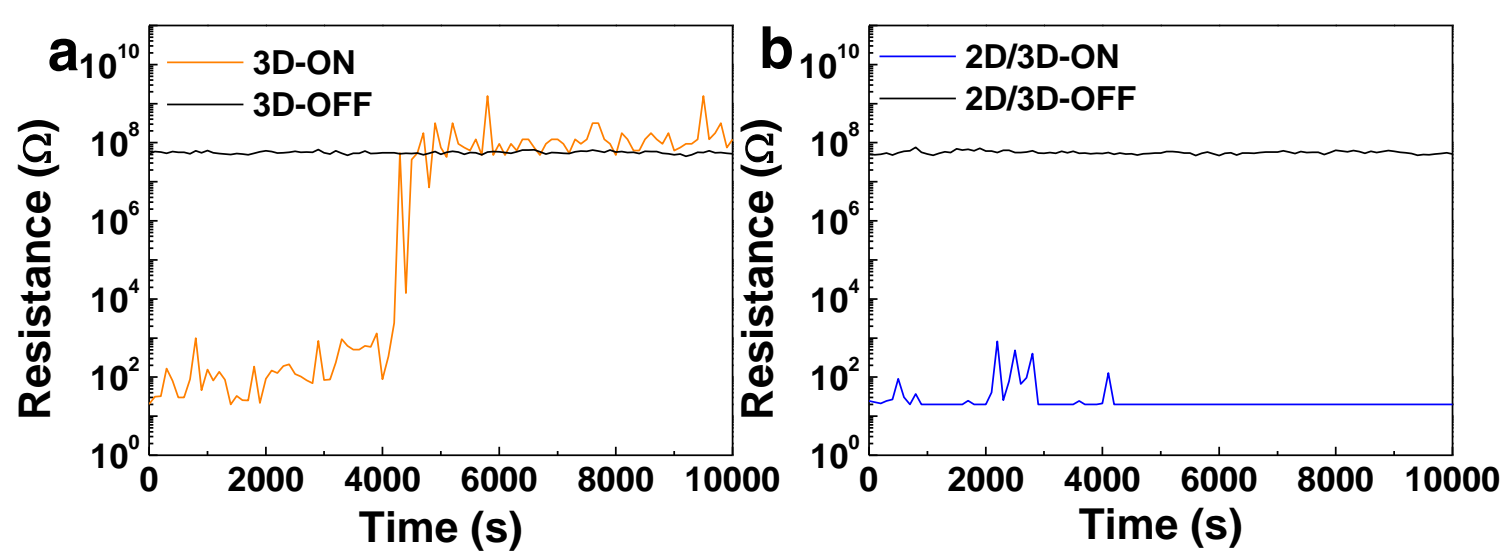

Figure S9. Retention characteristics of the HP-based RS devices: (a) 3D perovskite and (b) 2D/3D perovskite. 

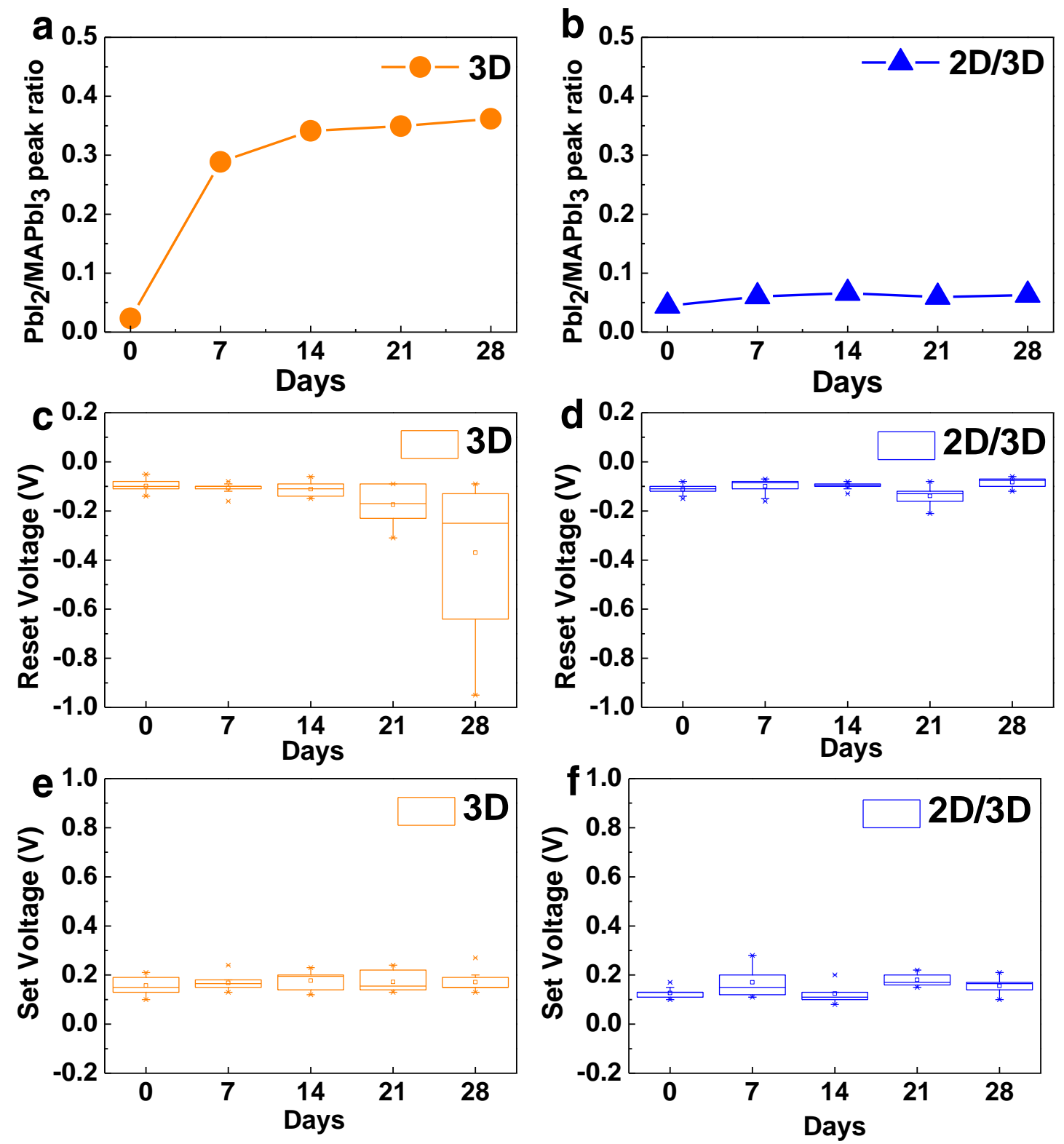

Figure S10. Long-term stability of the HP-based RS devices: (a,b) $\mathrm{PbI}_{2} / \mathrm{MAPbI}_{3}$ peak ratio of HP-based RS devices. (c,d) Reset voltage deviation of HP-based RS devices. (e,f) Set voltage deviation of HP-based RS devices. (a,c,e) 3D perovskite and (b,d,f) 2D/3D perovskite. 

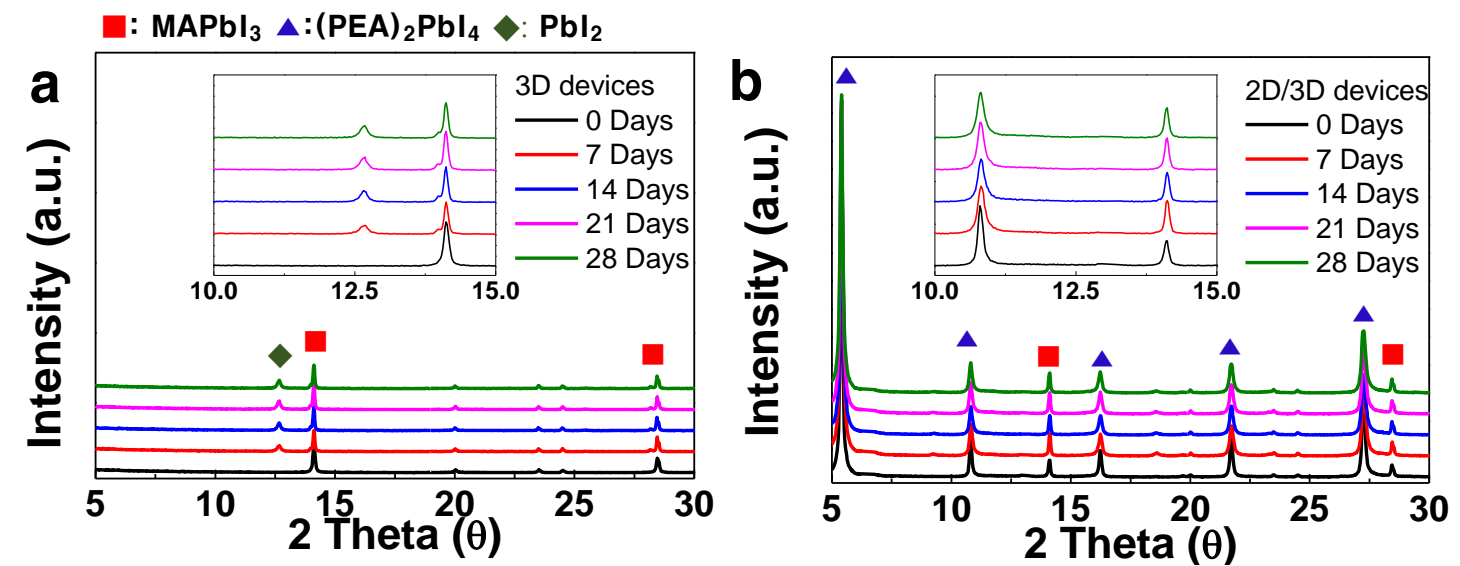

Figure S11. XRD patterns of the HP-based RS devices as a function of time: (a) 3D perovskite and (b) 2D/3D perovskite. 

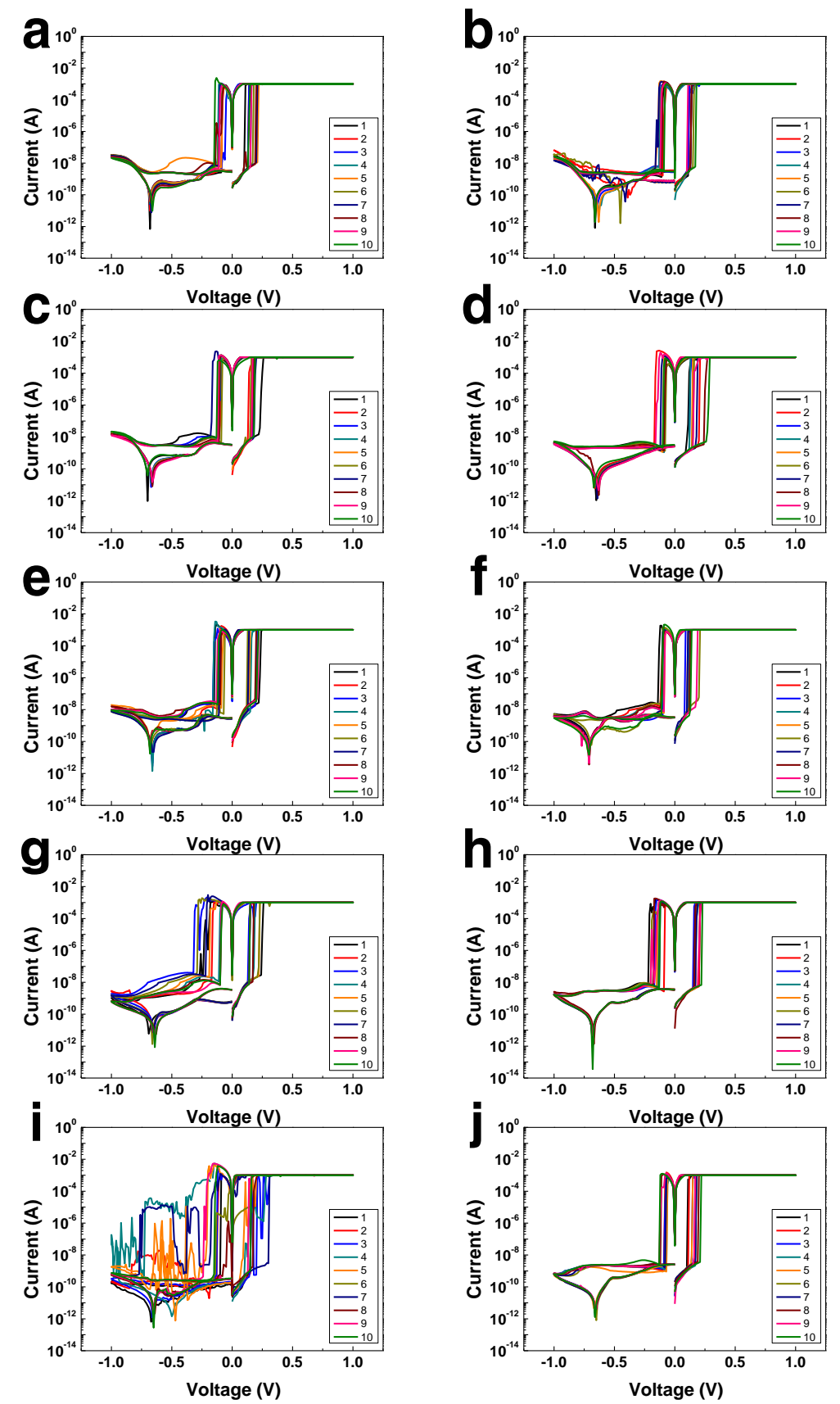

Figure S12. $I-V$ characteristics of HP-based RS devices for long-term stability: (a) 0 days 3D perovskite, (b) 0 days 2D/3D perovskite, (c) 7 days 3D perovskite, (d) 7 days 2D/3D perovskite, (e) 14 days 3D perovskite, (f) 14 days 2D/3D perovskite, (g) 21 days 3D perovskite, (h) 21 days 2D/3D perovskite, (i) 28 days 3D perovskite, and (j) 28 days 2D/3D perovskite. 
Table S1. Physical input parameters used for the COMSOL simulation.

\begin{tabular}{|lccc|}
\hline & $\begin{array}{c}\text { Thermal } \\
\text { Conductivity } \\
(\mathrm{w} / \mathrm{mK})\end{array}$ & $\begin{array}{c}\text { Heat Capacity } \\
(\mathrm{J} / \mathrm{kgK})\end{array}$ & Density $\left(\mathrm{g} / \mathrm{cm}^{3}\right)$ \\
\hline $\mathrm{Ag}$ & 406 & 233 & 10.5 \\
3D Perovskite & 0.28 & 333 & 4.16 \\
2D Perovskite & 1.18 & 559 & 1.58 \\
\hline
\end{tabular}

